\title{
Prevalence of Antinuclear Antibodies in Schoolchildren During Puberty and Possible Relationship with Musculoskeletal Pain: A Longitudinal Study
}

\author{
Francesca Sperotto, Giorgio Cuffaro, Sara Brachi, Mara Seguso, and Francesco Zulian
}

\begin{abstract}
Objective. The role of antinuclear antibodies (ANA) in children has still to be elucidated. The aim of our study was to evaluate the prevalence and persistence of ANA in schoolchildren during the puberty switch, and the possible relationship with chronic noninflammatory musculoskeletal pain (MSP).

Methods. Children aged 8-13 years and attending 4 public schools underwent a clinical examination, focusing on pubertal stage and presence of chronic noninflammatory MSP. Laboratory tests to determine the autoantibody-profile were also performed. Subjects with ANA positivity (titer $\geq 1: 80$ ) and/or chronic noninflammatory MSP were re-evaluated 3 years later.

Results. Two hundred sixty-one subjects enrolled in the study and $12.3 \%$ were ANA-positive, equally distributed in terms of sex and pubertal status. Three years later, in the group of patients studied for chronic noninflammatory MSP $(n=67)$, ANA positivity significantly increased from $13.4 \%$ to $44.8 \%$. In the ANA-positive cohort at baseline $(n=28), 92.9 \%$ of subjects were confirmed as being ANA-positive with a significantly increased titer. No association between ANA positivity and chronic noninflammatory MSP was found.

Conclusion. ANA prevalence and titers increase during puberty, especially in females, but have no relationship with chronic noninflammatory MSP. This finding may be related to the complex hormonal changes during the puberty switch period and opens new insights into autoimmunity. (First Release April 15 2014; J Rheumatol 2014;41:1405-8; doi:10.3899/jrheum.130948)
\end{abstract}

Key Indexing Terms:

ANTINUCLEAR ANTIBODIES PUBERTY MUSCULOSKELETAL PAIN

Antinuclear antibodies (ANA) are frequently found in pediatric patients with connective tissue diseases but may be triggered by infections, drugs, or a neoplastic process ${ }^{1,2}$ and have even been found in healthy individuals ${ }^{3,4,5,6}$. One study previously reported ANA positivity in association with noninflammatory musculoskeletal pain (MSP) in early childhood, but found no clinical relevance ${ }^{7}$.

The prevalence of positive ANA, reported in the literature for healthy individuals, ranges from $13.3 \%$, if we consider a titer $\geq 1: 80$, to $5.0 \%$ with a titer $\geq 1: 160^{3,4,7,8}$.

To date, few studies have addressed the role of ANA in healthy subjects, but no one has explored their frequency and meaning across the puberty switch, a period in which many autoimmune and connective tissue diseases develop ${ }^{9}$. The aim of our study was to evaluate the prevalence and persistence of ANA in subjects with no evident autoimmune

From the Department of Pediatrics, University of Padua, Padua, Italy.

F. Sperotto, MD, Pediatric Rheumatology Fellow; G. Cuffaro, MD, Pediatric Rheumatology Fellow; S. Brachi, MD, Pediatric Rheumatology Fellow; M. Seguso, MD, Assistant Professor, Laboratory Medicine; F. Zulian, MD, Assistant Professor, Rheumatology Unit, Department of Pediatrics, University of Padua.

Address correspondence to Dr. F. Zulian, Dipartimento di Pediatria,

Università di Padova, Via Giustiniani 3, 35128 Padua, Italy.

E-mail:zulian@pediatria.unipd.it

Accepted for publication January 8, 2014. disease and their possible relationship with chronic noninflammatory MSP over the course of puberty.

\section{MATERIALS AND METHODS}

In June 2009, we conducted a study on subjects attending 3 primary and 1 secondary state schools in the District of Padua, Italy, in order to evaluate the prevalence of chronic noninflammatory MSP, the prevalence of autoantibodies, and their reciprocal relationship.

Chronic MSP was defined as continuous or recurrent pain lasting more than 3 months and heavily interfering with daily activities, according to the International Association for the Study of Pain ${ }^{10}$. Each subject underwent a careful, general, and rheumatologic examination including the evaluation of the pubertal stage and the family history for autoimmune diseases in first degree relatives. The pubertal stage was assessed by the presence of secondary signs of pubertal development. For females, puberty was defined by breast development with a Tanner stage $\geq 3$ and menarche. For males, puberty was defined by testicles volume $\geq 12 \mathrm{ml}$ and evident pubic and underarm hair ${ }^{11,12}$.

Individuals with a history or the presence of neurologic, skeletal, metabolic, or autoimmune diseases were excluded to avoid a selection bias. Laboratory tests to determine the presence of ANA, extractable nuclear antigen antibodies (ENA), and anti-dsDNA were also performed. The ANA test consisted of the gold standard immunofluorescence antinuclear antibody test on HEp-2 cells (INOVA), using a fluorescein-conjugated anti-human immunoglobulin $\mathrm{G}(\mathrm{IgG})$ as a secondary antibody, according to the most recent position statement ${ }^{13,14}$. Samples were processed sequentially using a Zenit-plus analyzer and read by a NIKON fluorescence microscope and an LED-microscope by 2 independent operators. We considered positive a sample with titer $\geq 1: 80$. To increase sensitivity, sera

Personal non-commercial use only. The Journal of Rheumatology Copyright @ 2014 . All rights reserved. 
were also analyzed on triple murine tissue (kidney, stomach, liver; INOVA). The presence of ENA (SSA, SSB, Sm, RNP, Jo-1, Scl-70) was detected using the fluoride-immune-enzymatic method (PHADIA). Samples $>0.7$ were considered positive ${ }^{13}$. Anti-dsDNA was determined on a Crithidia luciliae substrate (INOVA) ${ }^{11}$.

Subjects with ANA positivity and/or chronic noninflammatory MSP were evaluated with the same methods 3 years later. Again, individuals with a history or the presence of neurologic, skeletal, metabolic, or autoimmune diseases were excluded, to avoid a further selection bias.

Demographic, clinical, and laboratory characteristics of patients were analyzed using descriptive statistics. Pearson's chi-square and Fisher's exact test were used to compare categorical variables between subgroups. Clinical variables obtained at baseline (2009) and 3 years later (2012) were compared using the Wilcoxon test and the McNemar test. A value of $\mathrm{p}<$ 0.05 was considered significant. The analysis was performed using StatsDirect statistical software (version 2.7.8, StatsDirect Ltd.).

The study was approved by the ethics committee of the Padua District Health Authority.

\section{RESULTS}

At baseline, blood tests were performed on 261/289 (90.3\%) subjects, aged 8-13 years old, with an F:M ratio of 1:1.1; $70.5 \%$ were prepubertal and $29.5 \%$ pubertal (Table 1). Thirty-two subjects (12.3\%) had ANA+ results, with the following distribution: $8.4 \%$ had 1:80 titer, $3.8 \% \geq 1: 160$, and $0.8 \% \geq 1: 320$ (Table 1 ). ANA positivity was equally distributed in terms of sex and pubertal status. None of the subjects that were ANA+ resulted in positive ENA or anti-dsDNA testing. A positive family history for autoimmune conditions in first-degree relatives was reported in $6.5 \%$ of the subjects, but no significant relationship with ANA positivity was found.
Three years later, in 2012, we re-evaluated the subjects with either chronic noninflammatory MSP at baseline ( $\mathrm{n}=$ 67) and/or ANA+ at baseline $(n=28)$. At followup, 7 patients overlapped between these 2 cohorts (Table 1).

ANA positivity, in the group of patients with previous chronic noninflammatory MSP, increased from $13.4 \%$ $(9 / 67)$ to $44.8 \%(30 / 67 ; \mathrm{p}<0.001)$, while no significant changes in the prevalence of positive family history for autoimmune diseases were found. Thirty-seven subjects $(55.2 \%)$ were persistently symptomatic, but there was no significant association with ANA. ANA positivity showed a trend that involved more prepubertal subjects $(59.1 \%)$ than pubertal ones (37.8\%) and more females (53.6\%) than males $(38.5 \%)$, but this was not statistically significant. In particular, ANA positivity involved more pubertal females than pubertal males $(50.0 \%$ vs $28.0 \%)$, while in the prepubertal period the prevalence was nearly equal between sexes.

The cohort of subjects previously ANA+ included 28 subjects whose demographic characteristics are summarized in Table 1 . The vast majority (92.9\%) of subjects with ANA+ at baseline were still positive at the 3-year followup evaluation. Two subjects decreased their titer from 1:80 to $1: 40$, but none became negative. ANA titer had the following distribution: $17.9 \%$ had 1:80 titer, $75.0 \% \geq 1: 160$, $50.0 \% \geq 1: 320$, and $14.3 \% \geq 1: 640$. Overall, autoantibodies titer showed a significant increase over time $(p=0.002)$, while the prevalence of positive family history for autoimmune diseases did not significantly change during the

Table 1. Demographic and clinical characteristics of schoolchildren according to the presence of antinuclear antibodies (ANA) or musculoskeletal pain (MSP).

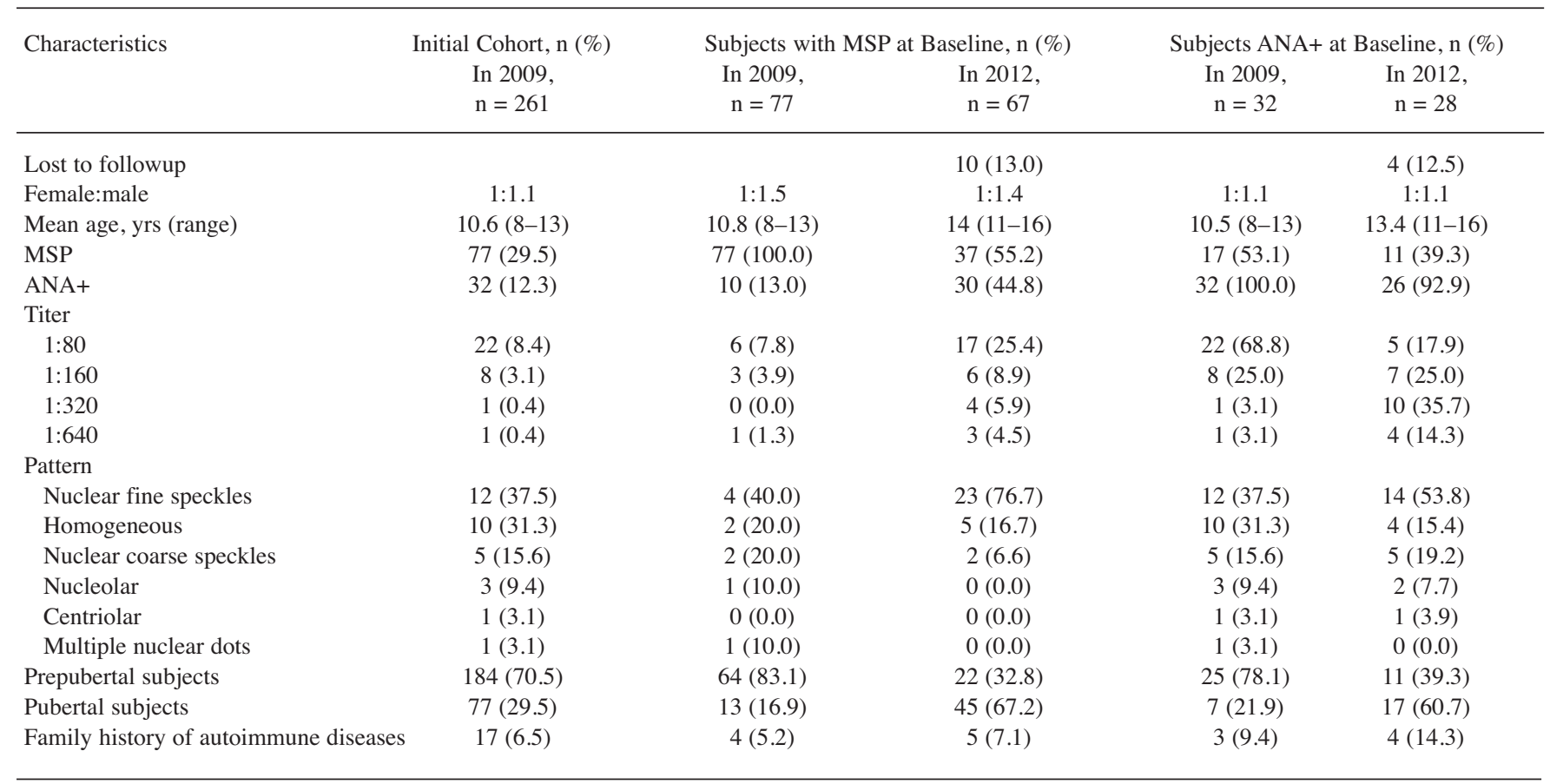


study period (Table 1). Eleven subjects (39.3\%) suffered from chronic noninflammatory MSP, but no significant association between ANA-positivity and symptoms was found.

The most frequent ANA-pattern found both at baseline and at followup was the "fine-speckled" as shown in Table 1.

\section{DISCUSSION}

It is well known that ANA frequently arise in the sera of children with autoimmune and connective tissue disease, including systemic lupus erythematosus, scleroderma, and juvenile idiopathic arthritis, but it is also present in organ-specific autoimmune diseases, such as autoimmune hepatitis and thyroiditis ${ }^{15,16}$ or during infectious processes, drug treatments or rarely, with malignancy ${ }^{1,2}$. ANA can also be found in healthy individuals, without any significant difference in prevalence for sex or age ${ }^{3,4,8}$. The prevalence of ANA reported in the general population ranges from $13.3 \%$ for a titer $\geq 1: 80$, to $3.3 \%$ for a titer $\geq 1: 320^{3}$. Studies in children reported similar or lesser prevalence ${ }^{4,8}$.

Although very few studies have addressed the issue of the role of ANA in healthy subjects, none has explored its frequency and meaning and across the puberty switch, a period in which onset occurs for many autoimmune and connective tissue diseases ${ }^{9}$.

In our study, we found a frequency of $12.3 \%$ ANA+ (titer $\geq 1: 80$ ) in healthy schoolchildren, equally distributed by sex and pubertal status. Indeed, the vast majority of these ANA+ subjects confirmed their ANA-positivity, with a significant increase of titer, 3 years later.

Despite this persistence and increased ANA titers in subjects transiting from the prepubertal to the pubertal stage, none developed autoimmune diseases. Previous studies reported similar results although the persistence of positive ANA was checked only 8 to 24 months after the first test ${ }^{6,17}$.

The cohort of children with chronic noninflammatory MSP at baseline showed an increased frequency of ANA positivity across puberty (from $13.4 \%$ to $44.8 \%$ ). This increased frequency was independent from the persistence of symptoms and confirmed the results of a previous study showing the absence of a significant association between ANA positivity and noninflammatory $\mathrm{MSP}^{7}$. On the other hand, a positive family history for autoimmune diseases does not seem to influence the prevalence of ANA during this period, because no significant statistical association was found.

As for the ANA pattern, fine speckled was the one most frequently found (Table 1). Although we have no precise definition on the subset of nuclear fine speckled pattern, several of the cases may correspond to the dense fine speckled pattern, which has been shown to consistently occur more frequently in healthy subjects rather than in those with autoimmune diseases ${ }^{18,19}$.

We do not have a clear explanation of why ANA positivity increases both in prevalence and titer across puberty. Clinical and experimental evidence supports the hypothesis that sex hormones modulate immunity. In fact, the majority of autoimmune diseases are more common in females than in males and, when stratified by age, their onset is more frequently around puberty ${ }^{9,20}$. Females tend to mount stronger reactions against infection than males and this is due to different factors such as genetics and imprinting elements, hormonal patterns, and cytokine profiles $^{20,21,22}$. In particular, the cytokines balance is responsible for determining the quality and direction of the immune response and females tend to show instability of this balance with predominantly proinflammatory TH1-mediated reactions as compared with males ${ }^{21,23}$. Our findings seem to be consistent with this evidence, because ANA were found more frequently in pubertal females than in males.

The hypothalamic-pituitary axis, whose activity starts around 4 years before puberty, also plays an important role in modulating the immune system ${ }^{9,24}$. Adenohypophyseal hormones seem to increase the differentiation and proliferation of $\mathrm{T}$ cells, making them more susceptible to antigenic stimulation ${ }^{9}$. The preadolescence period, in predisposed subjects, might stimulate an unspecific immune response resulting in ANA production.

The prevalence and titer of ANA increase across puberty, especially in females, but have no relationship with chronic noninflammatory MSP. None of the ANA-positive subjects developed rheumatic or autoimmune conditions over time. Further longterm prospective studies are needed to clarify the potential role of ANA as a marker of autoimmune-rheumatic conditions, particularly in the puberty switch period.

\section{REFERENCES}

1. Kaplan ME, Tan EM. Antinuclear antibodies in infectious mononucleosis. Lancet 1968;1:561.

2. Burnham TK. Antinuclear antibodies in patients with malignancies. Lancet 1972;2:436.

3. Tan EM, Feltkamp TE, Smolen JS, Butcher B, Dawkins R, Fritzler $\mathrm{MJ}$, et al. Range of antinuclear antibodies in healthy individuals. Arthritis Rheum 1997;40:1601-11.

4. Wananukul S, Voramethkul W, Kaewopas Y, Hanvivatvong O. Prevalence of positive antinuclear antibodies in healthy children. Asian Pac J Allergy Immunol 2005;23:153-7.

5. Volkmann ER, Taylor M, Ben-Artzi A. Using the antinuclear antibody test to diagnose rheumatic diseases: when does a positive test warrant further investigation? South Med J 2012;105:100-4.

6. Deane PM, Liard G, Siegel DM, Baum J. The outcome of children referred to a pediatric rheumatology clinic with a positive antinuclear antibody test but without an autoimmune disease. Pediatrics 1995;95:892-5.

7. Cabral DA, Petty RE, Fung M, Malleson PN. Persistent antinuclear antibodies in children without identifiable inflammatory rheumatic or autoimmune disease. Pediatrics 1992;89:441-4.

8. Hilario MO, Len CA, Roja SC, Terreri MT, Almeida G, Andrade LE. Frequency of antinuclear antibodies in healthy children and adolescents. Clin Pediatr 2004;43:637-42.

Personal non-commercial use only. The Journal of Rheumatology Copyright @ 2014 . All rights reserved. 
9. Athreya BH, Rettig P, Williams WV. Hypophyseal-pituitary-adrenal axis in autoimmune and rheumatic diseases. Immunol Res 1998;18:93-102.

10. Merskey H, Bogduk N. International Association for the Study of Pain. Classification of chronic pain: descriptions of chronic pain syndromes and definitions of pain terms. Seattle: IASP Press; 1994.

11. Marshall WA, Tanner JM. Growth and physiological development during adolescence. Ann Rev Med 1968;19:283-300.

12. Marshall WA, Tanner JM. Variation in the pattern of pubertal changes in girls. Arch Dis Child 1969;44:291-303.

13. Tozzoli R, Bizzaro N, Tonutti E, Villalta D, Bassetti D, Manoni F, et al. Italian Society of Laboratory Medicine. Guidelines for the autoantibody tests in the diagnosis and monitoring of autoimmune diseases. Am J Clin Pathol 2002;117:316-24.

14. Meroni PL, Schur PH. ANA screening: an old test with new recommendations. Ann Rheum Dis 2010;69:1420-2.

15. Petri M, Karlson EW, Cooper DS, Ladenson PW. Autoantibody tests in autoimmune thyroid disease: a case-control study. J Rheumatol 1991;18:1529.

16. Czaja AJ, Nishioka M, Morshed SA, Hachiya T. Patterns of nuclear immunofluorescence and reactivities to recombinant nuclear antigens in autoimmune hepatitis. Gastroenterology 1994;107:200.

17. Martini A, Lorini R, Zanaboni D, Ravelli A, Burgio RG. Frequency of autoantibodies in normal children. Am J Dis Child 1989;143:493-6.
18. Mariz HA, Sato EI, Barbosa SH, Rodrigues SH, Dellavance A, Andrade LE. Pattern on the antinuclear antibody-HEp-2 test is a critical parameter for discriminating antinuclear antibody-positive healthy individuals and patients with autoimmune rheumatic diseases. Arthritis Rheum 2011;63:191-200.

19. Mahler M, Parker T, Peebles CL, Andrade LE, Swart A, Carbone Y, et al. Anti-DFS70/LEDGF antibodies are more prevalent in healthy individuals compared to patients with systemic autoimmune rheumatic diseases. J Rheumatol 2012;39:2104-10.

20. Whitacre CC, Reingold SC, O'Looney PA. A gender gap in autoimmunity. Science 1999;283:1277-8.

21. Walker SE. The importance of sex hormones in lupus. In: Wallace DJ, Hahn GH, eds. Dubois' lupus erythematosus. 7th ed. Baltimore: Williams \& Wilkins; 2007:273-85.

22. Sandborg C. Expression of autoimmunity in the transition from childhood to adulthood: role of cytokines and gender. J Adolesc Health 2002;30:76-80.

23. Del Prete G. The concept of type- 1 and type- 2 helper $\mathrm{T}$ cells and their cytokines in humans. Int Rev Immunol 1998;16:427-55.

24. Chrousos GP. The hypothalamic-pituitary-adrenal axis and immune-mediated inflammation. N Engl J Med 1995;332:1351-62. 